\title{
Reflection mode acousto-optic imaging using a 1-D ultrasound array
}

Nowak, Lukasz, Steenbergen, Wiendelt

Lukasz J. Nowak, Wiendelt Steenbergen, "Reflection mode acousto-optic imaging using a 1-D ultrasound array," Proc. SPIE 11240, Photons Plus Ultrasound: Imaging and Sensing 2020, 1124020 (17 February 2020); doi: $10.1117 / 12.2544775$

SPIE. Event: SPIE BiOS, 2020, San Francisco, California, United States 


\title{
Reflection mode acousto-optic imaging using a 1-D ultrasound array
}

\author{
Lukasz J. Nowak ${ }^{\mathrm{a}}$ and Wiendelt Steenbergen ${ }^{\mathrm{a}}$ \\ ${ }^{a}$ Biomedical Photonic Imaging Group, Faculty of Science and Technology, University of \\ Twente, P.O. Box 217, 7500 AE, Enschede, The Netherlands
}

\begin{abstract}
Utilization of the acousto-optic effect by detection of light scattered within tissues and modulated by focused ultrasound pulses could provide diagnostic information impossible to obtain by purely acoustic or optical imaging modalities. It could also support photoacoustic imaging by mapping fluence rate distribution. However, practical implementation of this technique encounters numerous difficulties preventing it from rapid adoption in clinical use. One of the important limitations that has not yet been adequately addressed is that in many practical medical applications the region of interest may be accessed only from one side. In the present study we introduce the results of investigations on acousto-optic detection and localization of optically distinct inclusions inside acoustically homogeneous phantoms using a linear ultrasound array with electronically scanned focus and optical fibers arranged in reflectance geometry. Speckle contrast differences between speckle patterns captured in absence and presence of ultrasound pulses with different focal point coordinates are determined for various samples. The results allow clear distinction between phantoms with and without optically absorbing inclusions, although these are neither visible from the surface nor distinguishable on ultrasound images. It is also shown, that data analysis allows to obtain further cues on localization of absorbing regions. Conditions and limitations in this regard are discussed.
\end{abstract}

Keywords: Acousto-optic imaging, reflection mode, ultrasound, linear array transducer

\section{INTRODUCTION}

Acousto-optic imaging (AOI) is a technique that can potentially overcome the limitations of purely optical and acoustical medical imaging modalities by utilizing effects of interaction between focused ultrasound and light scattered in a tissue. Acoustic wave propagating through a medium causes periodic displacement of optical scatterers, and changes in resulting interference patterns of the detected light. Theoretical description of the underlying physical phenomena including various possible mechanisms of interaction was presented by Leutz ana Maret $^{1}$ and Wang et al. ${ }^{2}$ Recently several comprehensive reviews on acousto-optic imaging ${ }^{4-6}$ have been published.

Vast majority of the studies in the field concern transmission mode AOI. In such a configuration a light source and a detector are located on both sides of the investigated sample, while US transducer is placed perpendicularly to the transmitter-detector axis. This allows for more uniform illumination of the sample than in the reflection mode. Consequently, the detected changes in intensity or interference patterns of the detected light due to the change of focal point coordinates of US pulses will directly correspond to the changes in optical properties within the insonified volumes. Using this kind of setup, Lai et al. demonstrated possibility of imaging through $9,4 \mathrm{~cm}$ tissue-like phantom. ${ }^{7}$ Hussain et al. used for AOI six fiber bundles evenly distributed around a sample to transmit and receive light pulses. ${ }^{8}$ The US pulses were transmitted using a custom-made concave transducer array. They have determined fluence rate distribution of the collected light inside agar gel phantoms, ex-vivo tissue samples and freshly sacrificed mice.

Unfavorably, in many practical medical applications the region of interest may be accessed from one side only. Thus, light source, detector, and US transducer would have to be located next to each other. In such a reflection-mode configuration uniform illumination is no longer possible. Theoretical aspects of such an approach

Corresponding author: l.j.nowak@utwente.nl

Photons Plus Ultrasound: Imaging and Sensing 2020, edited by Alexander A. Oraevsky, Lihong V. Wang, Proc. of SPIE Vol. 11240, 112402O - @ 2020 SPIE · CCC code: 1605-7422/20/\$21 - doi: 10.1117/12.2544775 
were studied by Granot et al. ${ }^{9}$ The fluence rate of the collected light inside tissue or other highly scattering media will have a banana shaped distribution. Consequently, the measured changes in the detected light due to the different US focal point coordinates will be related not only to changes in optical properties between the insonified volumes, but also to the uneven fluence rate distribution inside these volumes. One of the solutions to overcome this issue might be to keep the relative positions of optical transmitter, receiver, and US transducer in fixed locations, while physically moving the investigated sample. This was done by Lev and Sfez, ${ }^{10-12}$ Hisaka and Sakura, ${ }^{13}$ Kim et al., ${ }^{14}$ and Hong-Bo et al. ${ }^{15}$ The studies demonstrated that it is possible to detect light absorbing inclusions inside phantoms and tissue samples using such a measurement configuration.

In the present study we introduce the results of investigations on reflection mode AOI using 1-D US array with electronically scanned focus. This approach allows for cross-sectional imaging while maintaining constant position of all the elements of the system and the medium, also without the need of using water tank for coupling. Thus, it is in this regard more feasible for in-vivo medical imaging than the other approaches described in the literature. Using electronically scanned focus in principle can also be significantly faster than physically moving the probe or a sample. The experimental investigations were carried out on acoustically homogeneous, opaque phantoms with tissue-like properties and optically distinct inclusions. We demonstrate that it is possible to detect and obtain cues on localization of those inclusions using the described setup.

\section{MATERIALS AND METHODS}

The experimental setup used for measurements is presented in Figure 1. A $532 \mathrm{~nm}$ continous wave (CW) laser with $6.5 \mathrm{~W}$ output power (Coherent Verdi 6) is used as a light source. An acousto-optic modulator (AOM)(Gooch \& Housego, R23080-3-LTD) is used to convert CW beam into $1 \mu$ s pulses triggered by an US scanner (Verasonics Vantage 256) through a gated $80 \mathrm{MHz}$ output signal driver (NEOS Technologies, 21080-2DM). The laser pulses are coupled into a $200 \mu \mathrm{m}$ multimode fiber connected to an integrated acousto-optic probe.

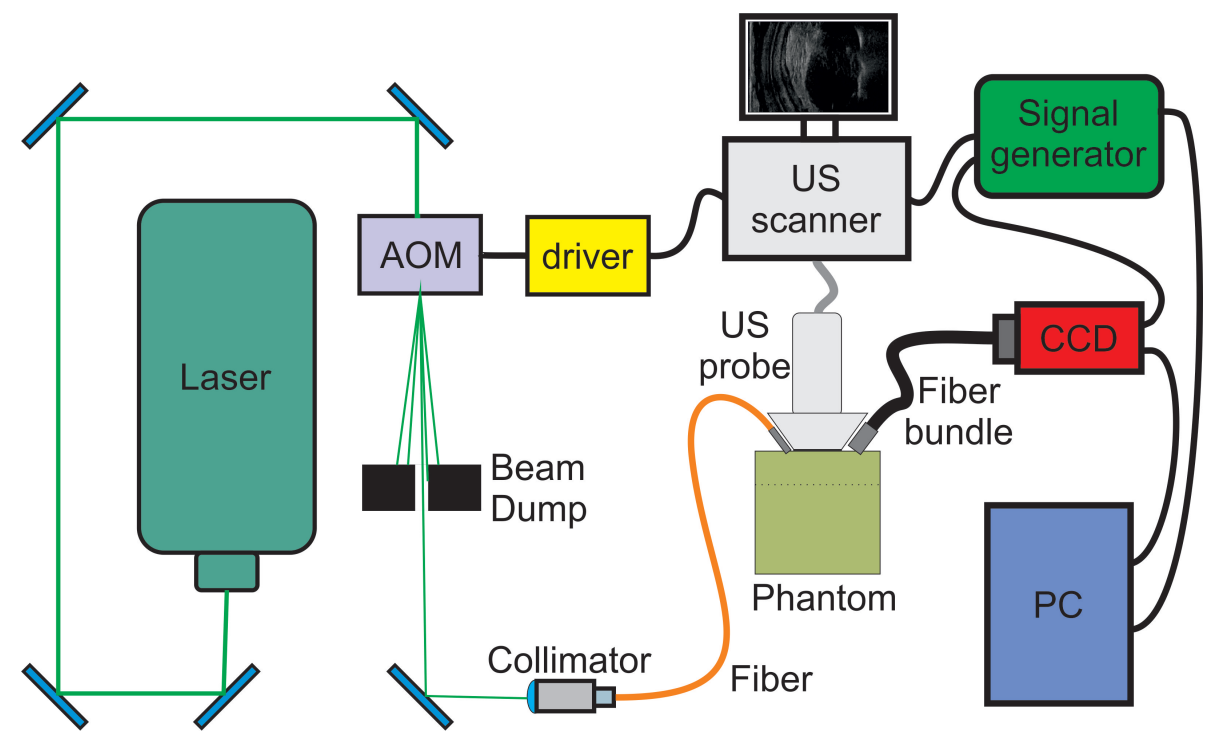

Figure 1. The experimental setup. AOM - acousto-optic modulator; CCD - charge-coupled device camera.

The integrated probe used for the measurements comprises the transmitting fiber, a 128 element US linear array (ATL L7-4), and a circular fiber bundle with a diameter of $4 \mathrm{~mm}$ for collecting light. All the components are held together by a custom made, 3D-printed enclosure. The fibers are at an angle of $20^{\circ}$ relative to the probe, inclined towards each other, $35 \mathrm{~mm}$ away, and oriented centrally and perpendicularly to the US transducer array. The integrated probe is pressed directly against the surfaces of the investigated samples. It is assumed that the middle point of the US array has coordinates $(x, y, z)=(0,0,0)$, and that the transducer elements are arranged along the $\mathrm{x}$ axis. The positive values of the $\mathrm{z}$ coordinates correspond to the sampling depth. 
The fiber bundle exit facet is positioned approximately $35 \mathrm{~mm}$ in front of the camera CCD array (Allied Vision Stingray F-125B), on which a speckle pattern is formed. The camera is triggered by an external signal generator with the frequency of $10 \mathrm{~Hz}$. The same triggering signal is fed to the US scanner, which further triggers the laser. The laser pulses are delayed relative to the US pulses by a time period determined independently for every single focal point in such a way that the illumination starts when the acoustic wave reaches its focus. The camera is connected to a computer for data acquisition.

For every captured frame we determine the speckle contrast value $C$ defined as: ${ }^{16}$

$$
C=\frac{\sigma}{\langle I\rangle},
$$

where $\sigma$ stands for standard deviation of the pixel values, and $\langle I\rangle$ is their mean value. Acoustic wave propagating inside an irradiated, diffusive medium interacts with the light by inducing vibrations of the optical scatterers and refractive index changes. The resulting light modulation will lead to a decrease in the observed speckle contrast. This decrease is proportional to the acoustic power. ${ }^{2,16}$

The signal to noise ratio is in general very low, as the fraction of the modulated and detected light is also low. Thus, all of the studies in the field employ averaging over several tens or even thousands of frames. We have captured 250 frame pairs for every single imaging point. The exposure time was $50 \mathrm{~ms}$, during which 50 laser pulses (accompanied with the synchronized US pulses for every second frame) were transmitted.

The experimental investigations on AOI were carried out on cuboid phantoms with dimensions of $700 \times 480 \times 350 \mathrm{~mm}^{3}$. The phantoms were casted from polyvinyl chloride plastisol (PVCP, Lure Flex firm by Lure Factors) with addition of $3,5 \frac{\mathrm{mg}}{\mathrm{ml}}$ of titanium dioxide (Aldrich, particle size $44 \mu \mathrm{m}$ ) for optical scattering. The acoustic and optical properties of PVCP can be suited to approximate the properties of different human tissues ${ }^{17,18}$ Our goal was to develop acoustically homogeneous, opaque phantoms with optically distinct inclusions. The phantoms were casted in two-step processes. First, hot PVCP was poured into a cubical mold with a cylindrical metal bar inside. The bar was stretched centrally between opposite walls of the mold, and removed from the phantom after curing. Next, the same PVCP material with addition of black ink (Lure Factors, std. black) was heated and injected into the remaining hole inside the PVCP block. Phantoms without inclusions were also casted as a reference for measurements. The inclusions inside phantoms were neither visible on US images nor from the surface. The propagation velocity of acoustic waves inside phantoms was determined to be $1700 \frac{\mathrm{m}}{\mathrm{s}}$. Optical properties of the PVCP were determined based on spectrophotometer measurements (Shimadzu UV-2600) using inverse adding-doubling method. ${ }^{19}$ Optical scattering coefficient $\mu_{s}$ was determined to be approximately $4.5 \mathrm{~cm}^{-1}$. Absorption coefficient $\mu_{a}$ was found to be negligible for PVCP without ink, and equal to approximately $17 \mathrm{~cm}^{-1}$ for the same material with ink. Phantoms with inclusions of different sizes, oriented along different edges, and positioned at various depths were casted.

\section{RESULTS}

Speckle contrast difference (SCD) values were determined for different phantoms and various probe positions relative to the inclusions. The speckle contrast value in the absence of US varied between different measurements and was in the range of approximately 0.4-0.52. Figure 2 presents SCD values as functions of US focal point coordinates obtained for two different phantoms: one without any inclusion, and the other one with $4 \mathrm{~mm}$ diameter inclusion $11 \mathrm{~mm}$ below the surface (center of the cylinder), oriented during the measurements along $\mathrm{Y}$ axis (i.e. perpendicularly to the US transducer array and along the optical transmitter-detector axis). The probe was positioned at the center point, above the inclusion. The SCD values obtained for the phantom with the inclusion are in every case lower. The differences are the most significant for smaller focusing depths.

Figure 3 presents the comparison of SCD values obtained for the same phantom with $4 \mathrm{~mm}$ diameter inclusion $11 \mathrm{~mm}$ below the surface, oriented along $\mathrm{Y}$ axis, for two different probe positions: directly above the inclusion, and displaced $6 \mathrm{~mm}$ along the $\mathrm{X}$ axis. Thus, in the latter case the inclusion was parallel and $6 \mathrm{~mm}$ off the optical transmitter-detector axis. As it can be seen, moving the probe aside results in increase of the obtained SCD values within the whole considered range of the US focus coordinates. Again, the differences are the most significant for smaller focusing depths. 


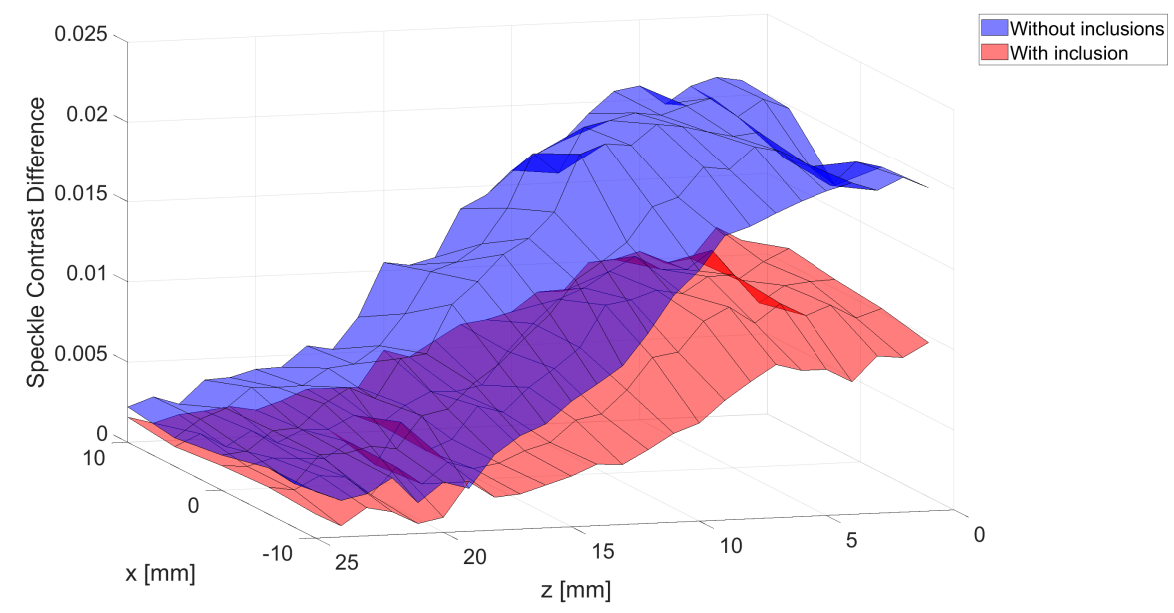

Figure 2. Speckle contrast difference values as functions of US focal point coordinates for phantoms without any inclusion and with $4 \mathrm{~mm}$ diameter inclusion $11 \mathrm{~mm}$ below the surface.

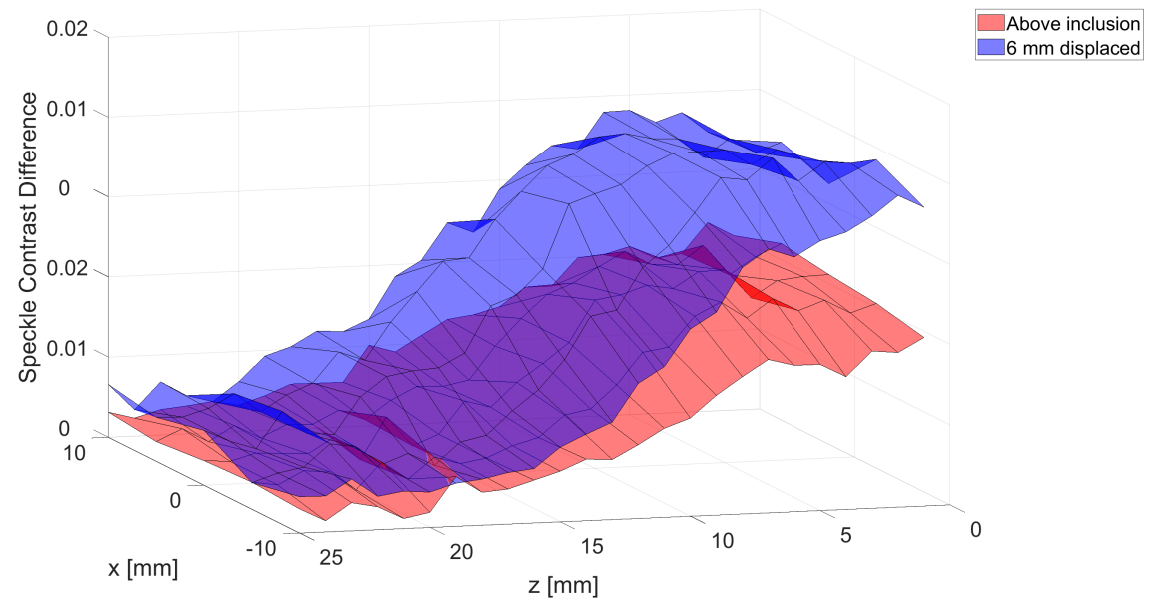

Figure 3. Speckle contrast difference values as functions of US focal point coordinates determined for phantom with 4 mm diameter inclusion $11 \mathrm{~mm}$ below the surface and two probe positions: directly above the inclusion, and $6 \mathrm{~mm}$ displaced.

The results presented in Figures 2 and 3 allow for clear distinction between the considered phantoms and relative probe-inclusion positions based on the obtained SCD values. However, this is only possible by comparison of different datasets. In order to exploit the benefits of electronic scanning of US focus the information should be extracted directly from the results of measurements gathered without physically moving the probe. Here, we observe changes in the SCD values distribution occurring within the whole range of the considered US focal point coordinates, and not only locally. To address this issue, we have investigated the symmetry deviations of the SCD distribution on the XZ plane in the case when an inclusion was positioned off the center axis of the probe $(\mathrm{x}=0, \mathrm{y}=0)$. We define an asymmetry coefficient $A C_{\chi}$ as:

$$
A C_{\chi}(z)=S C D(\chi, z)-S C D(-\chi, z)
$$

where $S C D(\chi, z)$ and $S C D(-\chi, z)$ denote speckle contrast difference values determined for US focal points coordinates $(x=\chi, z)$, and $(x=-\chi, z)$, respectively.

Figure 4 presents examples of $A C_{5}(z)$ values determined for phantoms without any inclusion and with $4 \mathrm{~mm}$ diameter inclusion $11 \mathrm{~mm}$ below the surface, oriented along $\mathrm{Y}$ axis, for two different probe positions: at the center point of a phantom, and $6 \mathrm{~mm}$ aside (the inclusion was in this case oriented along the line $(\mathrm{x}=6 \mathrm{~mm}$, 
$\mathrm{z}=11 \mathrm{~mm})$ ). In the case of the optically homogeneous phantom, no significant differences are visible between the results obtained for various probe positions, which fluctuate around zero. The results are also similar when an inclusion is present directly below the probe. However, if the inclusion is moved off the center axis of the probe, then a significant drop (or peak, depending on the direction) in the determined $A C_{5}(z)$ values is observed. The location of the minimum is related to the actual depth of the absorbing region inside the phantom.
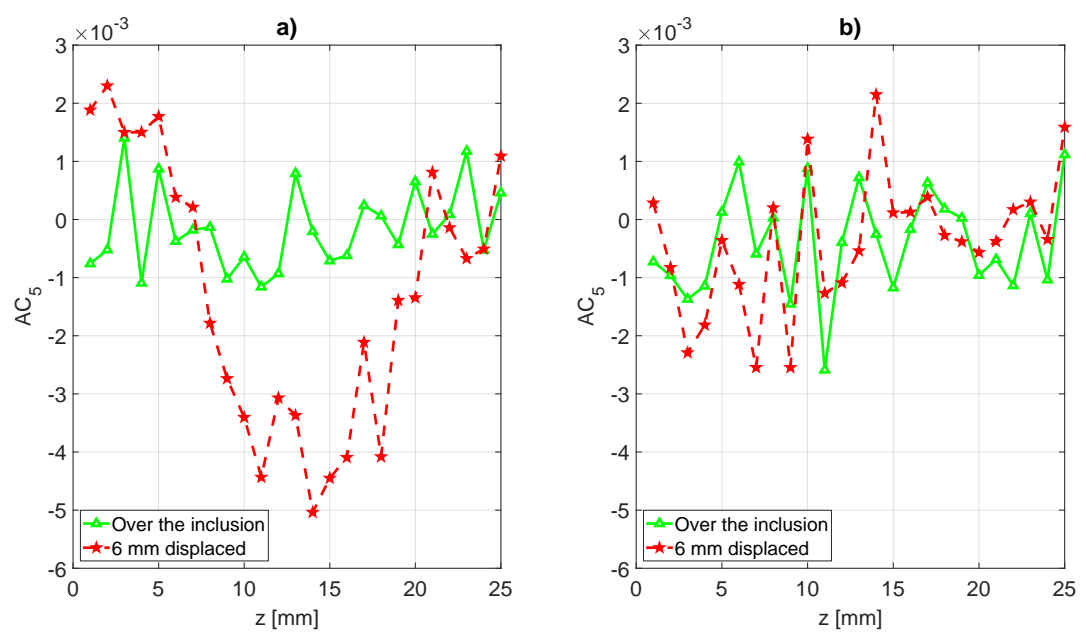

Figure 4. Asymmetry coefficient $A C_{5}$ as a function of imaging depth, determined for phantoms with 4 mm diameter inclusion $11 \mathrm{~mm}$ below the surface, oriented along $\mathrm{Y}$ axis (a), and without any inclusions (b), for two different probe positions: at the center point of a phantom, and $6 \mathrm{~mm}$ aside.

In the results presented in Figures 2 and 3 the absorbing inclusion is not visible by a distinct local dip in SCD distributions. We hypothesized that it should be possible to observe the local minima of the SCD values in some specific cases of sufficiently small absorbing inclusions localized within regions of relatively high illumination. To test this hypothesis, we have performed measurements on a phantom with a $2 \mathrm{~mm}$ diameter inclusion 5,5 $\mathrm{mm}$ below the surface, oriented along $\mathrm{x}$ axis - i.e. in parallel to the US array and perpendicularly to the optical transmitter-detector axis. The orientation of inclusion was chosen this way in this specific case in order to minimize the total amount of the absorbed light. The SCD values obtained while scanning US focal point along the $\mathrm{z}$ axis are presented in Figure 5. The probe was located directly above the inclusion. A distinct local minimum in the SCD is clearly visible, albeit at a larger range of z-values than the inclusion.

\section{DISCUSSION AND CONCLUSIONS}

The present study hypothesizes that it is possible to detect and potentially also localize light absorbing inclusions inside acoustically homogeneous, opaque samples using a reflection mode AOI system with 1-D linear US array. To test this claim we have developed various phantoms fulfilling the assumed conditions and we have determined the SCD values for different US focal point coordinates. The hypothesis holds in the light of the presented results of measurements. In all the investigated cases the presence of an inclusion in a region below the probe manifests itself by a significant SCD drop, compared to results obtained for the phantom without any inclusions, as shown in Figure 2. Changing the relative position or orientation of the probe and an inclusion also results in changes in the observed SCD values, as presented in Figure 3.

In the reflection mode AOI both optical transmitter and detector have to be positioned on the same side of the sample, thus the fluence rate distribution of the detected light is highly non-uniform in a general case. It follows that for a fixed probe position the SCD values determined as a function of US focal point coordinates will reveal certain variability, even for an optically homogeneous sample. This can be clearly seen in Figure 2 . This phenomenon constitutes the biggest difference between the described approach with electronically steered US focus and other studies in which either the whole integrated probe or the investigated sample are physically displaced. In the latter case the detected differences in SCD values can be directly translated to changes in 


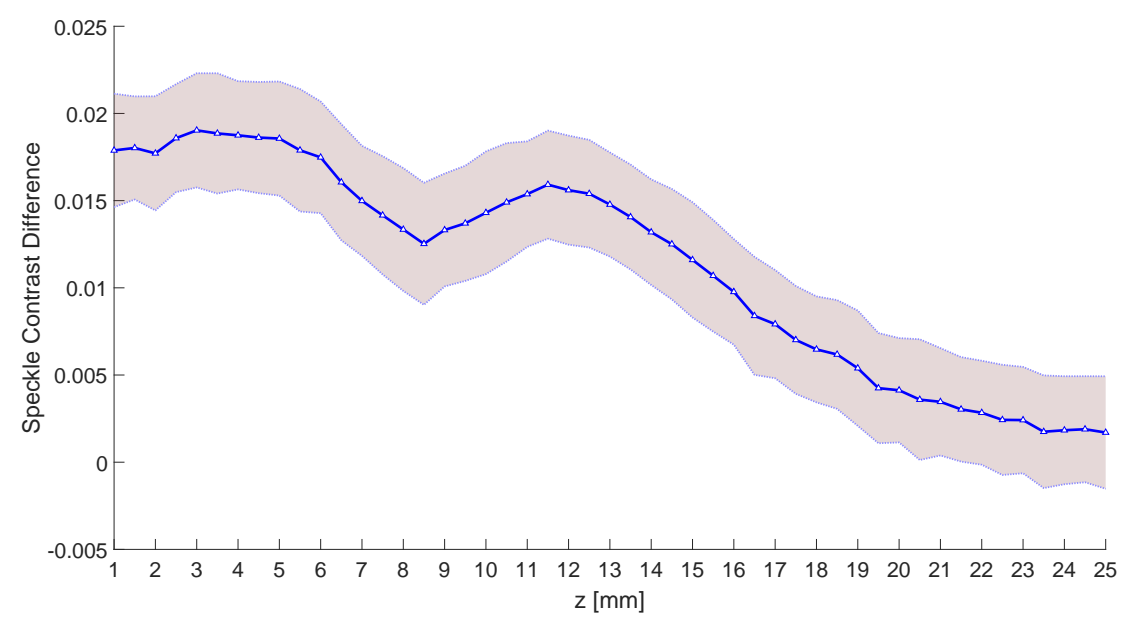

Figure 5. Speckle contrast difference values obtained for a phantom with $2 \mathrm{~mm}$ diameter inclusion $5,5 \mathrm{~mm}$ below the surface, oriented along $\mathrm{X}$ axis. The US focal point was scanned along $\mathrm{Z}$ axis, while its $\mathrm{x}$ coordinate was 0 .

optical properties within the illuminated and insonified volume (assuming no significant changes to the acoustic pressure field distribution, which is the common approach).

The results presented in Figures 2 and 3 allow to conclude that it is possible to detect an optically absorbing inclusion based on comparison between measurements taken for different samples or probe positions. Such an approach, however, does not allow to exploit benefits emerging from the electronic US focus steering. For this reason we have investigated the symmetry deviations of the determined SCD values in the case when an inclusion is positioned off the optical transmitter-detector axis. We have introduced the asymmetry coefficient $A C_{\chi}$, defined with equation (2). The results of measurements indicate that analysis of $A C_{\chi}$ values can provide important cues not only for detection, but also localization of optically absorbing regions within an investigated sample. This has been illustrated on the example of $A C_{5}$ plots as functions of the US focusing depth, presented in Figure 4. In the case of phantom without any inclusions or with an inclusion located directly below the probe, the determined $A C_{5}$ values fluctuate around zero. If the probe is displaced and the investigated sample does not reveal symmetry about the optical axis anymore, then a significant local extremum can be observed. The type of the extremum (in the presented case - minimum) indicate if the inclusion is to the left or to the right of the center of the probe. The location of the minimum corresponds to the actual depth of the inclusion, with additional extension towards greater $\mathrm{z}$ values.

A distinctive feature that could potentially be used for detection and localization of optically absorbing regions within investigated samples is the presence of local minima on the determined SCD value plots. For the adopted illumination scheme with single transmitter and detector such phenomena should not occur in the case of optically homogeneous samples. Although we did not observe such minima on the plots presented in Figures 2 and 3, we have hypothesized that it should be possible for some special cases of inclusion size and geometry. We decided to cast another phantom with smaller inclusion located closer to the surface, and to orient it along $\mathrm{X}$ axis, i.e. perpendicularly to the optical transmitter-detector axis. The intention was to minimize the total amount of the absorbed light and to allow formation of more distinct local maxima of the fluence rate distribution of the detected light below the inclusion than in the case of previously used samples. In experiments we have used a phantom with a $2 \mathrm{~mm}$ diameter inclusion, located $5,5 \mathrm{~mm}$ below the surface. In this case, the dip on the determined SCD profile is clearly visible.

\section{ACKNOWLEDGMENTS}

The study was funded by grant 15228 of NWO Division Applied and Engineering Sciences and the Dutch Cancer Society. 


\section{REFERENCES}

[1] Leutz, W. and Maret, G., "Ultrasonic modulation of multiply scattered light," Physica B: Condensed Matter 204, 14-19 (Jan. 1995).

[2] Wang, L. V., "Mechanisms of ultrasonic modulation of multiply scattered coherent light: a Monte Carlo model," Optics Letters 26, 1191-1193 (Aug. 2001).

[3] Yao, G. and Wang, L. V., "Theoretical and experimental studies of ultrasound-modulated optical tomography in biological tissue," Applied Optics 39, 659-664 (Feb. 2000).

[4] Elson, D. S., Li Rui, Dunsby Christopher, Eckersley Robert, and Tang Meng-Xing, "Ultrasound-mediated optical tomography: a review of current methods," Interface Focus 1, 632-648 (Aug. 2011).

[5] Resink, S. G., Steenbergen, W., and Boccara, A. C., "State-of-the art of acoust-optic sensing and imaging of turbid media," Journal of Biomedical Optics 17, 040901 (Apr. 2012).

[6] Gunther, J. and Andersson-Engels, S., "Review of current methods of acousto-optical tomography for biomedical applications," Frontiers of Optoelectronics 10, 211-238 (Sept. 2017).

[7] Lai, P., Xu, X., and Wang, L. V., "Ultrasound-modulated optical tomography at new depth," Journal of Biomedical Optics 17, 066006 (June 2012).

[8] Hussain, A., Hondebrink, E., Staley, J., and Steenbergen, W., "Photoacoustic and acousto-optic tomography for quantitative and functional imaging," Optica 5, 1579-1589 (Dec. 2018).

[9] Granot, E., Lev, A., Kotler, Z., Sfez, B. G., and Taitelbaum, H., "Detection of inhomogeneities with ultrasound tagging of light," JOSA A 18, 1962-1967 (Aug. 2001).

[10] Lev, A., Kotler, Z., and Sfez, B. G., "Ultrasound tagged light imaging in turbid media in a reflectance geometry," Optics Letters 25, 378-380 (Mar. 2000).

[11] Lev, A. and Sfez, B. G., "Direct, noninvasive detection of photon density in turbid media," Optics Letters 27, 473-475 (Apr. 2002).

[12] Lev, A. and Sfez, B., "In vivo demonstration of the ultrasound-modulated light technique," JOSA $A$ 20, 2347-2354 (Dec. 2003).

[13] Hisaka, M. and Sasakura, Y., "Light Scattering Characteristics of Biological Tissues in Coaxial UltrasoundModulated Optical Tomography," Japanese Journal of Applied Physics 48, 067002 (June 2009).

[14] Kim, C., Song, K. H., Maslov, K. I., and Wang, L. V., "Ultrasound-modulated optical tomography in reflection mode with ring-shaped light illumination," Journal of Biomedical Optics 14, 024015 (Mar. 2009).

[15] Hong-Bo, F., Da, X., Ya-Guang, Z., Yi, W., and Qun, C., "Ultrasound-Modulated Optical Tomography in Reflective and Coaxial Configuration," Chinese Physics Letters 20, 2165-2168 (Dec. 2003).

[16] Li, J., Ku, G., and Wang, L. V., "Ultrasound-modulated optical tomography of biological tissue by use of contrast of laser speckles," Applied Optics 41, 6030 (Oct. 2002).

[17] Vogt, W. C., Jia, C., Wear, K. A., M.d, B. S. G., and Pfefer, T. J., "Biologically relevant photoacoustic imaging phantoms with tunable optical and acoustic properties," Journal of Biomedical Optics 21, 101405 (Feb. 2016).

[18] Dantuma, M., Dommelen, R. v., and Manohar, S., "Semi-anthropomorphic photoacoustic breast phantom," Biomedical Optics Express 10, 5921-5939 (Nov. 2019).

[19] Prahl, S., [Optical Property Measurements using the Inverse Adding-Doubling Program], Oregon Medical Laser Center, St. Vincent Hospital (1999). 